\title{
Infectious Mononucleosis as a Cause of Severe Pseudothrombocytopenia
}

\author{
Marina Llopis ${ }^{\mathrm{a}}$, Nuria Yague ${ }^{\mathrm{b}}$, Inmaculada Poquet ${ }^{\mathrm{a}}$, Carlos Tornero $^{\mathrm{a}, \mathrm{c}}$
}

\begin{abstract}
Infectious mononucleosis (IM) is characterized by fever, swallowing pain (with or without pharyngeal exudate), neck adenopathies and, in characteristic cases, atypical lymphocytosis. Over $50 \%$ of all patients with IM present generally mild thrombocytopenia without clinical consequences, though in exceptional cases the condition may prove serious. Pseudothrombocytopenia (PT) is an in vitro artifact caused by antibody (generally IgG) - mediated platelet aggregation secondary to blood sample collection and processing in a medium containing ethylenediaminetetraacetic acid (EDTA). It can be associated to autoimmune disorders, drugs, solid tumors, and myeloproliferative and lymphoproliferative syndromes. We present a case of PT associated to IM in which the viral infection was the cause of false severe thrombopenia.
\end{abstract}

Keywords: Infectious mononucleosis; Pseudothrombocytopenia; Etiology

\section{Introduction}

Infectious mononucleosis (IM) is characterized by fever, swallowing pain (with or without pharyngeal exudate), neck adenopathies and, in characteristic cases, atypical lymphocytosis. Different conditions have been associated with IM, and although over $80 \%$ of all cases can be attributed to acute Epstein-Barr virus (EBV) infection, other possible etiologies are cytomegalovirus (CMV), primary human immunodefi-

\footnotetext{
Manuscript accepted for publication May 6, 2014

${ }^{a}$ Internal Medicine Department, Hospital Francesc de Borja, Paseo Germanias 71, 46702 Gandia (Valencia), Spain

${ }^{\mathrm{b}}$ Hmatology Department, Hospital Francesc de Borja, Paseo

Germanias 71, 46702 Gandia (Valencia), Spain

${ }^{\mathrm{c}}$ Corresponding Author: Carlos Tornero, Internal Medicine

Department, Hospital Francesc de Borja, Paseo Germanias 71, 46702

Gandia (Valencia), Spain. Email: tornero_car@gva.es
}

doi: http://dx.doi.org/10.14740/jmc1826e ciency virus (HIV) infection, acute Toxoplasma gondii infection, and infections produced by human herpes simplex virus (HSV) 6 [1]. Over $50 \%$ of all patients with IM present generally mild thrombocytopenia without clinical consequences, though in exceptional cases the condition may prove serious [2].

Pseudothrombocytopenia (PT) is an in vitro artifact caused by antibody (generally IgG, but also IgA and IgM) - mediated platelet aggregation secondary to blood sample collection in a medium containing ethylenediaminetetraacetic acid (EDTA). EDTA-mediated PT is the most common cause of PT, with an incidence of 1/1,000 cytometric tests [3].

\section{Case Report}

We present a patient with PT associated to IM in which the viral infection was the cause of false severe thrombopenia. Such cases have rarely been described in the literature $[4,5]$.

A 16-year-old female was admitted to our department with neck adenopathies during the last week, accompanied by asthenia, pharyngeal discomfort and local pain. The laboratory tests revealed thrombopenia $(30,000-50,000 /$ $\mathrm{mm}^{3}$ ), though the blood smears showed platelet aggregates together with reactive lymphocytes typical of IM (Fig. 1).

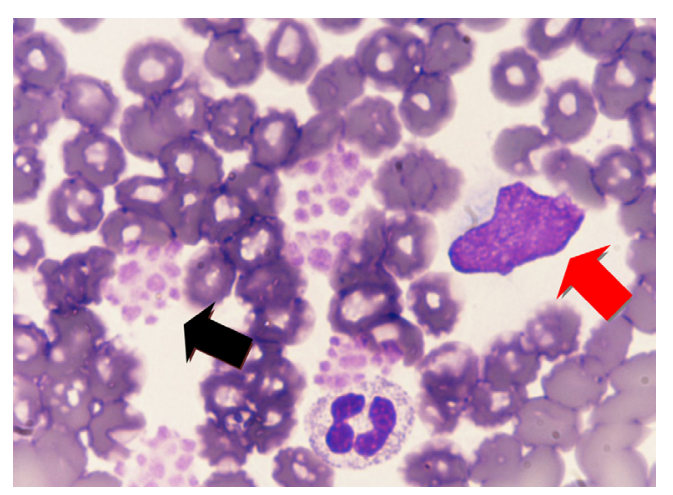

Figure 1. Platelet aggregates are observed (black arrows), together with reactive lymphocytes typical of infectious mononucleosis (red arrows). 
IM was therefore diagnosed and confirmed by the presence of IgM against EBV, with seroconversion 1 month later of IgG against EBV viral capsid antigen. During admission, the laboratory tests made in citrated tubes with manual counts yielded a platelet count of $>120,000 / \mathrm{mm}^{3}$. One month later, control testing in tubes containing EDTA revealed a platelet count of $132,000 / \mathrm{mm}^{3}$, with no platelet aggregates in the smears.

\section{Discussion}

IM can cause thrombocytopenia through autoimmune mechanisms in over $50 \%$ of the cases, though the drop in platelet count is usually mild and asymptomatic, and requires no treatment.

PT has been related to blood sample collection and processing in a medium containing EDTA, and can be associated to autoimmune disorders, drugs, solid tumors, and myeloproliferative and lymphoproliferative syndromes. The cause of the reaction with EDTA appears to be related to glycoprotein IIb/IIIa (platelet fibrinogen receptor) [6, 7]. The explanation for the platelet aggregates would be a change in the structure of the glycoprotein, with the expression of new surface antigens that would be targeted by certain antibodies. This conformational change of the protein is produced by the chelating action of EDTA, since the presence of calcium is needed to maintain the structure of the glycoprotein [7, 8]. In addition to a drop in platelet count evidenced by the auto-analyzer, other laboratory test alterations can also be seen, such as pseudoleukocytosis and platelet "satellitism" phenomena. Pseudoleukocytosis is a false increase in leukocyte count in which the auto-analyzer counts the platelet aggregates as leukocytes, while "satellitism" is characterized by platelet adherence to the periphery of the neutrophils observed in the peripheral blood smear [7].

We have described a case in which IM was the direct cause of PT, giving rise to false severe thrombopenia. Such cases have rarely been described in the literature [4, 5], though the possibility of such situations must be taken into account in order to avoid diagnostic and management errors.

\section{References}

1. Godshall SE, Kirchner JT. Infectious mononucleosis. Complexities of a common syndrome. Postgrad Med. 2000;107(7):175-179, 183-174, 186.

2. Fica A. Sindrome de mononucleosis infecciosa en pacientes adolescentes y adultos. Rev Chil Infect. 2003;20(4):235-242.

3. Payne BA, Pierre RV. Pseudothrombocytopenia: a laboratory artifact with potentially serious consequences. Mayo Clin Proc. 1984;59(2):123-125.

4. Hsieh AT, Chao TY, Chen YC. Pseudothrombocytopenia associated with infectious mononucleosis. Arch Pathol Lab Med. 2003;127(1):e17-18.

5. Choe WH, Cho YU, Chae JD, Kim SH. Pseudothrombocytopenia or platelet clumping as a possible cause of low platelet count in patients with viral infection: a case series from single institution focusing on hepatitis A virus infection. Int J Lab Hematol. 2013;35(1):70-76.

6. Fiorin F, Steffan A, Pradella P, Bizzaro N, Potenza R, De Angelis V. IgG platelet antibodies in EDTA-dependent pseudothrombocytopenia bind to platelet membrane glycoprotein IIb. Am J Clin Pathol. 1998;110(2):178183.

7. Savage RA. Pseudoleukocytosis due to EDTA-induced platelet clumping. Am J Clin Pathol. 1984;81(3):317322.

8. Fuster Soler JL, Bermudez Cortes M, Gutierrez Macias A, Ibanez Garcia JM y Martinez Lopez JL. Seudotrombocitopenia dependiente de EDTA. Acta Pediatr Esp. 2002;60:275-277. 